\title{
A Spatial Supply-Demand Imbalance Between Rural Primary and Secondary Schools in Shaanxi Province Based on Road Network Analysis
}

\author{
Ziwei Qiang, Bei Jin*, Shiyuan Xu \\ College of Economics \& Management, Northwest A\&F University, Xi'an 710119, China \\ *Corresponding author: Bei Jin, jinbei@nwsuaf.edu.cn
}

Copyright: (C) 2022 Author(s). This is an open-access article distributed under the terms of the Creative Commons Attribution License (CC BY 4.0), permitting distribution and reproduction in any medium, provided the original work is cited.

\begin{abstract}
It is of great significance to optimize the spatial matching of basic education facilities in rural areas and promote the equalization of urban and rural basic public services and the overall revitalization of rural areas. Based on the theory of spatial accessibility and spatial balance of supply and demand, this paper constructs the evaluation system of rural basic education facilities and layout rationality. In this study, we took 42 primary and secondary schools and 2089 rural areas with concentrated population in six township areas of Hancheng City as the research subjects. We used ArcGIS network analysis, population coupling model, and other methods, from the administrative scale and rural population concentration area scale for evaluation. The accessibility of basic education facilities and its coupling relationship with the spatial distribution of student population describe the spatial and geographical pattern of basic education facilities in rural areas. The results show that the accessibility of rural administrative scale and the spatial balance of supply and demand of population concentration area scale are associated with great problems, the differences between towns are obvious, and the overall level of the best performing towns is still low. On the scale of population concentration area, the overall coupling degree is too low, the "dislocation" distribution of educational facilities and student population is obvious, and the matching degree of the student population and school in different towns is significantly different, showing a more obvious "core-periphery" spatial structure characteristics centered on the eastern and southeast regions. The terrain characteristics of rural areas in Shaanxi province are prominent, and the accessibility of the road network is particularly significant. Therefore, optimizing the construction of rural roads and optimizing school layout according to local conditions will become an important solution to promote the balanced allocation of basic education resources in Shaanxi province.
\end{abstract}

Keywords: Rural basic education facilities; Network analysis; Balanced spatial layout; Spatial population coupling

Online publication: January 20, 2022

\section{Introduction}

Since the reform and opening-up, China has begun to transition from a subsistence-oriented society with the main task of solving food and clothing issues to a development-oriented society to promote all-around human development. The overall rapid growth of public demand throughout society and the lack of public services and shortage of basic public products have become prominent contradictions in the new stage of transformation. The main contradiction of Chinese society in the new era has been transformed into the contradiction between people's growing need for a better life as well as unbalanced and inadequate development, which is most prominently reflected in the rural areas. The Strategic Plan for the Revitalization of Rural Areas (2018-2022) emphasizes the need to "promote public education, health care, 
social security, and other resources to rural areas, gradually establish a sound basic public service system that covers all people, is universally shared and integrated between urban and rural areas, and promote the equalization of basic public services in urban and rural areas."

The "No. 1 Document" clearly states that it will "accelerate the mending of the shortcomings of the rural habitat and public services" and "comprehensively improve the level of public services such as education, healthcare, social security, pensions, culture, and sports in rural areas, and accelerate the equalization of basic public services in urban and rural areas, which is initiated and driven by the government. There is an urgent need to improve public services in rural areas. As rural education is an important part of rural infrastructure, optimizing the allocation of education facilities in rural primary and secondary schools to further promote the construction of rural public service facilities and realize the equalization of basic public services between urban and rural areas has become an important task in the new stage of coordinating urban and rural development, promoting urban-rural integration and implementing the rural revitalization strategy.

Based on this, this study took 42 primary and secondary schools in six township areas of Hancheng City with 2089 rural population clusters as the research subjects, breaking the traditional supply-based basic education research model as the starting point, while taking into account the main subject of education resource balance, i.e., students. Using ArcGIS network analysis and population coupling model, the study measured and evaluated the accessibility of primary schools to provide a scientific basis for bridging the shortcomings of rural development and implementing the rural revitalization strategy from the perspective of promoting balanced education development.

\section{Theoretical foundations}

\subsection{Spatial accessibility}

The concept of accessibility was first introduced by Hansen in 1959, who defined the concept of accessibility as the magnitude of opportunities for nodes in a transportation network to interact with each other ${ }^{[1]}$. Accessibility, as an important indicator of the functional efficiency and spatial equity of regional spatial structures, has been widely used in fields such as regional development and transport planning ${ }^{[2,3]}$. Meanwhile, in the field of public service facilities research, the spatial accessibility evaluation method can identify the scarcity of public services in these areas, which is a measure of the layout of public services and facilities space equity and is an effective way to measure the layout of facilities ${ }^{[4,5]}$. The spatial accessibility can be evaluated from two perspectives ${ }^{[3]}$. Accessibility can be divided into four categories based on two perspectives (explicit and implicit, spatial and non-spatial). Researchers are more interested in implicit accessibility, where planners and policymakers can evaluate the existing state of the service system and seek strategies to improve services based on the implicit accessibility of certain types of services in an area ${ }^{[6]}$. Spatial accessibility focuses on the distance barrier linking supply and demand points or the transport medium to overcome this barrier, while non-spatial accessibility focuses on non-geographical elements ${ }^{[7]}$. This paper focuses on the issue of implicit spatial accessibility.

\subsection{Balanced spatial supply and demand}

Since the industrial revolution in the $18^{\text {th }}$ century, the rapid development of industrialization and urbanization has raised the level of global development, but it has also brought negative impacts such as widening regional development gaps and uncontrolled spatial order. The huge differences in natural conditions and economic bases between regions and the basic national conditions of rapid industrialization and urbanization have led to a series of deep-seated problems such as imbalanced regional development, disorderly spatial development, and a prominent urban-rural dual structure in China, which are particularly 
serious issues ${ }^{[8]}$. The long-standing neglect of long-term scientific planning of spatial layout is an important cause of spatial imbalance in China ${ }^{[9]}$. The long-standing neglect of long-term scientific planning of spatial layout is a major cause of spatial imbalance in China.

The study of spatial equilibrium started earlier in foreign countries, and its development has gone through regional division of labor. The development of spatial equilibrium has gone through three stages: regional division of labor ${ }^{[10]}$, location choice ${ }^{[11,12]}$, and spatial equilibrium under the new economic geography ${ }^{[11,13]}$. In China, scholars have mostly focused on "three-dimensional equilibrium," i.e., social, economic, and ecological values in geographical latitudes, for a comprehensive consideration of spatial equilibrium ${ }^{[14,15]}$. This spatial equilibrium implies that the population, economy, and ecology of the region are in balance. This spatial equilibrium implies a spatially optimal state of "Pareto efficiency" in which population, economy, resources, and environment are coordinated, i.e., the optimal spatial allocation of economic goods and activities ${ }^{[16,17]}$. The spatial equilibrium implies a state of optimal spatial allocation of economic goods and activities. The fundamental contradiction in the construction of public infrastructure and services is that of the imbalance between supply and demand. This is reflected in the spatial equilibrium of the state, i.e., the "Pareto state" of space. "Supply" and "demand" in economic theory correspond to "land" and "people" in layout planning, i.e., the optimal spatial allocation of economic and social resources and environment is formed by matching the supply capacity of the spatial supply subject - "land" - with the demand capacity of the spatial demand subject - "people." ${ }^{[18]}$ The spatial allocation of economic and social resources and environment is formed by matching the supply capacity of the spatial supply subject "land" - with the demand capacity of the spatial demand subject - "people."

\section{Research data and methodology}

\subsection{Study area and data}

The city of Hancheng $\left(110^{\circ} 07^{\prime} 19^{\prime \prime}-110^{\circ} 37^{\prime} 24^{\prime \prime} \mathrm{E}, 35^{\circ} 18^{\prime} 50^{\prime \prime}-35^{\circ} 52^{\prime} 08^{\prime \prime} \mathrm{N}\right)$ is located on the west bank of the Yellow River in eastern Shaanxi Province, in the northeastern corner of the Guanzhong Basin, and is a planned city within Shaanxi Province. Hancheng's topography is high in the northwest and low in the southeast. The deep mountains in the west are mostly beam-like hills, the shallow mountains in the middle are mostly loess hills and the loess plateau in the east. The 108 national highway runs through the north and south, the city and township roads are popular, and the two horizontal and one vertical national and provincial highways form the main skeleton of the highway in Hancheng, namely the Xiyu Expressway and the G108 national highway, and the one vertical provincial highway S304.

The data in this paper mainly includes data on rural primary and secondary school sites in Hancheng City, traffic and road network data, and demographic data. The original data on student population and township population and area were obtained from Hancheng Yearbook, Shaanxi Regional Statistical Yearbook, and China County Statistical Yearbook, and verified through internet inquiries, telephone consultations, and field research. Spatial data such as the distribution of residential land was obtained by vectorization of the administrative map of Hancheng prepared by the Bureau of Land and Resources of Hancheng.

\subsection{Research methodology}

\subsubsection{Network analysis}

Network analysis, as an important part of GIS spatial analysis, is a spatial analysis method to simulate and analyze network structure and resource allocation based on the construction of network datasets and to geographically analyze and model geographic networks and urban infrastructure networks to explore spatial optimization solutions ${ }^{[19]}$. It is a method of geographic analysis and modeling of geographical networks 
and urban infrastructure networks to explore spatial optimization solutions. The network service area represents the range of areas within a specified impedance, i.e., the 15-min service area of a point represents the range of all areas that can be reached within 15 min from that point ${ }^{[20]}$. The network service area is the area within the specified impedance range. The use of network analysis tools to assess accessibility can lead to more reasonable results, so network analysis is used in the real-time road ${ }^{[21]}$. It is used in a wide range of applications such as navigation, urban network design and management, and the layout of public services.

\subsubsection{Spatial demographic coupling analysis}

The spatial coupling degree can reflect the degree of matching and coordination of elements within a system and the strength of interaction and influence between systems or elements ${ }^{[22]}$. The spatial coupling degree can reflect the degree of matching and coordination of elements within a system and the strength of interaction and influence between the system or elements. The basic unit of concentration of rural population is the residential land, so this study used each residential land to represent the population concentration area and calculates the population weight of each residential land using the floor area of residential land. The population weight of each residential area was calculated using the interpolation method of area weights, using the floor area of each residential area as the basic weight and the town as the source area to obtain the population weight of each population agglomeration, and the number of people owned by each residential area was obtained according to the weight coefficient. Then, the basic model of coupling degree of any residential place $\mathrm{i}$ is expressed as follows:

$$
C_{i}=P \times \frac{d_{\max }}{d_{i}}=\frac{\frac{A_{i}}{A_{j}} \times P_{j}}{P_{\max }} \times \frac{d_{\max }}{d_{i}}
$$

where $C_{i}$ denotes the spatial coupling between settlement $\mathrm{i}$ and a school facility; $\mathrm{P}$ is the population weight of students in settlement $i$; $d_{i}$ is the distance from the nearest school facility within a radius of $3 \mathrm{~km}$ from the center of $\mathrm{i} ; d_{\max }$ is the distance from the residential area to the farthest school facility within a radius of $3 \mathrm{~km} ; A_{i} / A_{j}$ is the ratio of the floor area of settlement $i$ to the sum of the floor areas of all the settlements in commune $\mathrm{j} ; P_{j}$ is the student population of township $\mathrm{j} ; P_{\max }$ is the population of the settlement with the highest weight of students in the rural area of Hancheng (the population weight is measured as the ratio of the area of the settlements, i.e. $\left.A_{i} / A_{j}\right)$. Regarding $C_{i}$, the larger the coupling, the better the coupling, and vice versa.

\section{Analysis of results}

\subsection{Accessibility analysis}

According to field research, rural primary school students tend to walk while middle school students tend to cycle to school. This paper is based on the traffic road network in Hancheng City, combined with existing research results on five road grades given different walking and cycling speeds as shown in Table 1. The speed of each road class was designed concerning the "Technical Standards for Highway Engineering of the People's Republic of China (JTGB01-2014)" and was used as the basis for setting the walking and cycling speeds for primary and secondary school students on different road classes. The effective reachable time for primary schools was set at $15 \mathrm{~min}, 30 \mathrm{~min}$, and $45 \mathrm{~min}$, i.e., the farthest service distance is controlled at $1000 \mathrm{~m}, 2000 \mathrm{~m}$, and $3000 \mathrm{~m}$, respectively, based on a walking speed of $4 \mathrm{~km} / \mathrm{h}$ and a cycling speed of $10 \mathrm{~km} / \mathrm{h}$. The effective reachable time for secondary schools was set at $10 \mathrm{~min}, 15 \mathrm{~min}$, and 30 
min, i.e., the farthest service distance was controlled at $1000 \mathrm{~m}, 2000 \mathrm{~m}$, and $3000 \mathrm{~m}$. The farthest service distance was controlled at $1700 \mathrm{~m}, 2500 \mathrm{~m}$, and $5000 \mathrm{~m}$, combined with the road network for the effective reachable range analysis.

Table 1. Composition and speed of the road network

\begin{tabular}{cccccccc}
\hline Road grade & Highways & $\begin{array}{c}\text { Class I } \\
\text { roads }\end{array}$ & $\begin{array}{c}\text { Secondary } \\
\text { roads }\end{array}$ & $\begin{array}{c}\text { Tertiary } \\
\text { roads }\end{array}$ & $\begin{array}{c}\text { Class IV } \\
\text { roads }\end{array}$ & Outside & Remarks \\
\hline Walking & 2 & 5 & 5 & 5 & 4 & 4 & Primary schools \\
Cycling & 4 & 15 & 15 & 12 & 12 & 10 & Secondary schools \\
\hline
\end{tabular}

Unit: $\mathrm{km} / \mathrm{h}$

Hancheng's middle school accessibility is slightly better than elementary schools. The eastern part of the city has relatively good coverage of basic education resources, the southern part is the next best, while the northern and western parts of the city have a wide range of blind spots. This is related to the distribution of schools and road networks due to the topography, with the west being mostly mountainous areas with few schools and less accessible roads of lower density and grade. The densely populated areas are mainly located in the eastern part of the city, where there are more schools and better access to roads of higher density and grade. In particular, the effective reachable service coverage of primary and secondary schools in Longmen Town and Xizhuang Town is greater, and the effective reachable range of secondary school cycling in Zhichuan Town and Zhiyang Town performs better compared to primary schools. There is a positive correlation between the coverage and the population density and the degree of economic development.

Specifically, rural primary schools in Hancheng City served 6.71, 20.62, and 42.52 km at 15, 30, and $45 \mathrm{~min}$, respectively, accounting for $0.44 \%, 1.34 \%$, and $2.77 \%$ of the total study area. The area served by rural secondary schools in Hancheng City at $10 \mathrm{~min}, 15 \mathrm{~min}$, and $30 \mathrm{~min}$ was $16.39,32.56$, and $85.55 \mathrm{~km}^{2}$ respectively, accounting for $1.07 \%, 2.12 \%$, and $5.57 \%$ of the total area of the study area, respectively; none of which reached $10 \%$. This is strongly related to the wide distribution of mountainous areas in the study area. The ratio of accessibility to the township area for primary and secondary schools in each township in Hancheng City is shown in Table 2. The service area ratio for rural primary schools in each township for a 45-min walk is below 50\%, with the highest being Longmen Township (31.69\%), followed by Xizhuang Township (5.79\%) and Zhichuan Township (3.37\%), while Banqiao Township (0.71\%), Zhiyang Township $(0.66 \%)$ and Sanshuping Township $(0.10 \%)$ have service area ratios of less than $1 \%$, which is related to the large area of their townships and their mostly mountainous terrain. Only Longmen Township (54.31\%) has a 30-min area ratio for secondary school rides of over 50\%, Xizhuang Township (11.24\%) reaches $10 \%$, Zhichuan Township (7.06\%) and Zhiyang Township (6.24\%) are similarly above 5\%, Banqiao Township $(2.36 \%)$, and Sangshuping Township is less than $1 \%(0.82 \%)$. 
Table 2. Effective reachable area and proportion by township

\begin{tabular}{ccccccccc}
\hline & $\begin{array}{c}\text { Primary } \\
\text { school by } \\
\text { walking }(\mathrm{min})\end{array}$ & $\begin{array}{c}\text { Effective } \\
\text { reachable } \\
\text { area }\left(\mathrm{km}^{2}\right)\end{array}$ & $\begin{array}{c}\text { Township } \\
\text { area } \\
\left(\mathrm{km}^{2}\right)\end{array}$ & $\begin{array}{c}\text { Area } \\
\text { ratio } \\
(\%)\end{array}$ & $\begin{array}{c}\text { Secondary } \\
\text { school by } \\
\text { cycling }(\mathrm{min})\end{array}$ & $\begin{array}{c}\text { Effective } \\
\text { reachable } \\
\text { area } \\
\left(\mathrm{km}^{2}\right)\end{array}$ & $\begin{array}{c}\text { Townshi } \\
\text { p area } \\
\left(\mathrm{km}^{2}\right)\end{array}$ & $\begin{array}{c}\text { Area } \\
\text { ratio } \\
(\%)\end{array}$ \\
\hline \multirow{2}{*}{ Longmen } & $0-15$ & 2.8247 & 65 & 4.35 & $0-10$ & 8.1538 & 65 & 12.54 \\
Township & $0-30$ & 9.7433 & 65 & 14.99 & $0-15$ & 15.2644 & 65 & 23.48 \\
& $0-45$ & 20.5956 & 65 & 31.69 & $0-30$ & 35.3017 & 65 & 54.31 \\
\hline \multirow{2}{*}{ Xizhuang } & $0-15$ & 2.7612 & 241.5 & 1.14 & $0-10$ & 5.7296 & 241.5 & 2.37 \\
Township & $0-30$ & 6.9495 & 241.5 & 2.88 & $0-15$ & 11.2208 & 241.5 & 4.65 \\
& $0-45$ & 13.9735 & 241.5 & 5.79 & $0-30$ & 27.1477 & 241.5 & 11.24 \\
\hline \multirow{2}{*}{ Zhichuan } & $0-15$ & 0.5772 & 182 & 0.32 & $0-10$ & 0.6331 & 182 & 0.35 \\
Town & $0-30$ & 1.9201 & 182 & 1.05 & $0-15$ & 1.5534 & 182 & 0.85 \\
& $0-45$ & 6.1386 & 182 & 3.37 & $0-30$ & 12.8535 & 182 & 7.06 \\
\hline \multirow{2}{*}{ Banqiao } & $0-15$ & 0.1561 & 292 & 0.05 & $0-10$ & 0.4495 & 292 & 0.15 \\
Town & $0-30$ & 0.6397 & 292 & 0.22 & $0-15$ & 1.2725 & 292 & 0.44 \\
& $0-45$ & 2.0643 & 292 & 0.71 & $0-30$ & 6.8827 & 292 & 2.36 \\
\hline \multirow{2}{*}{ Zhiyang } & $0-15$ & 0.0739 & 173 & 0.04 & $0-10$ & 2.3126 & 173 & 1.34 \\
Township & $0-30$ & 0.3391 & 173 & 0.20 & $0-15$ & 3.9383 & 173 & 2.28 \\
& $0-45$ & 1.1388 & 173 & 0.66 & $0-30$ & 10.7955 & 173 & 6.24 \\
\hline \multirow{2}{*}{ Sangshuping } & $0-15$ & 0.0526 & 582.5 & 0.01 & $0-10$ & 0.2795 & 582.5 & 0.05 \\
Township & $0-30$ & 0.1903 & 582.5 & 0.03 & $0-15$ & 0.6485 & 582.5 & 0.11 \\
& $0-45$ & 0.5556 & 582.5 & 0.10 & $0-30$ & 4.7508 & 582.5 & 0.82 \\
\hline \multirow{2}{*}{ Towny }
\end{tabular}

\subsection{Spatial supply and demand equilibrium analysis}

The matching of the distribution of rural primary and secondary school facilities to the residential area is an important indicator of the level of supply and demand, and the layout of schools must not only have an appropriate catchment area but also take into account the degree of coupling with the actual spatial distribution of the population. In the previous analysis, the effective reach of the supply side of educational resources - schools - was derived and the level of supply of rural primary and secondary schools was analyzed. To further the level of demand satisfaction, i.e., how well the layout of rural primary and secondary school facilities matches the population of students living there, this section uses a spatialdemographic coupling degree model for further analysis. The coupling degree between each population catchment area and school distribution was calculated through equation (1), and then spatially interpolated through ArcGIS's kriging interpolation method to obtain a schematic diagram of the spatial coupling between the primary and secondary school student population and primary and secondary schools.

As shown in Figure 1, there is a significant variation in the degree of matching between student population and schools in different towns and villages in Hancheng, showing a clear "core-periphery" spatial structure centered on the eastern and southeastern regions. This may be due to the high topography in the northwest and low topography in the southeast of Hancheng. The western part of the city is mostly deep in the mountains, with sparse settlements and few schools. The eastern part of the city is mainly a loess plateau, which is suitable for living and has a dense distribution of schools and settlements, with a relatively good degree of coupling. 
The relatively high degree of coupling is concentrated in the northern part of Longmen Town and the central part of Zhichuan Town, while the degree of coupling in other peripheral areas decreases from the northern part of Longmen Town and the central part of Zhichuan Town to the periphery in order. The areas with a lower coupling degree are concentrated in the town of Sanshuping, the western part of Xizhuang town, Banqiao town, and the western part of Ziyang town show a significantly decreasing trend towards the western edge. Figure 1 shows that a distinct low-density area with a blind area appears in the eastern part of Hancheng City appears in the southeastern part of Xizhuang Town, because this area is the area through which the Yellow River flows, and the blind area that appears in the eastern part of Longmen Town is also due to the flow of the Yellow River. The blind areas in the north-western part of Longmen Town are due to the absence of settlements in the area near the administrative boundary. This is because the central part of the city is flatter than the western part and therefore has a higher density of residential areas than the western part, but the schools in the central part of the city are not well configured so there are no accessible schools within $3 \mathrm{~km}$ of some of the residential areas, indicating that there is a large area of "misaligned" distribution of educational facilities and population. The best coupling between population and educational facilities is 0.42 in the northeastern part of Longmen Town. This area has a high density of student population and a large number of schools, so the spatial distribution of schools and students is relatively well-matched, but still does not exceed 0.50 .
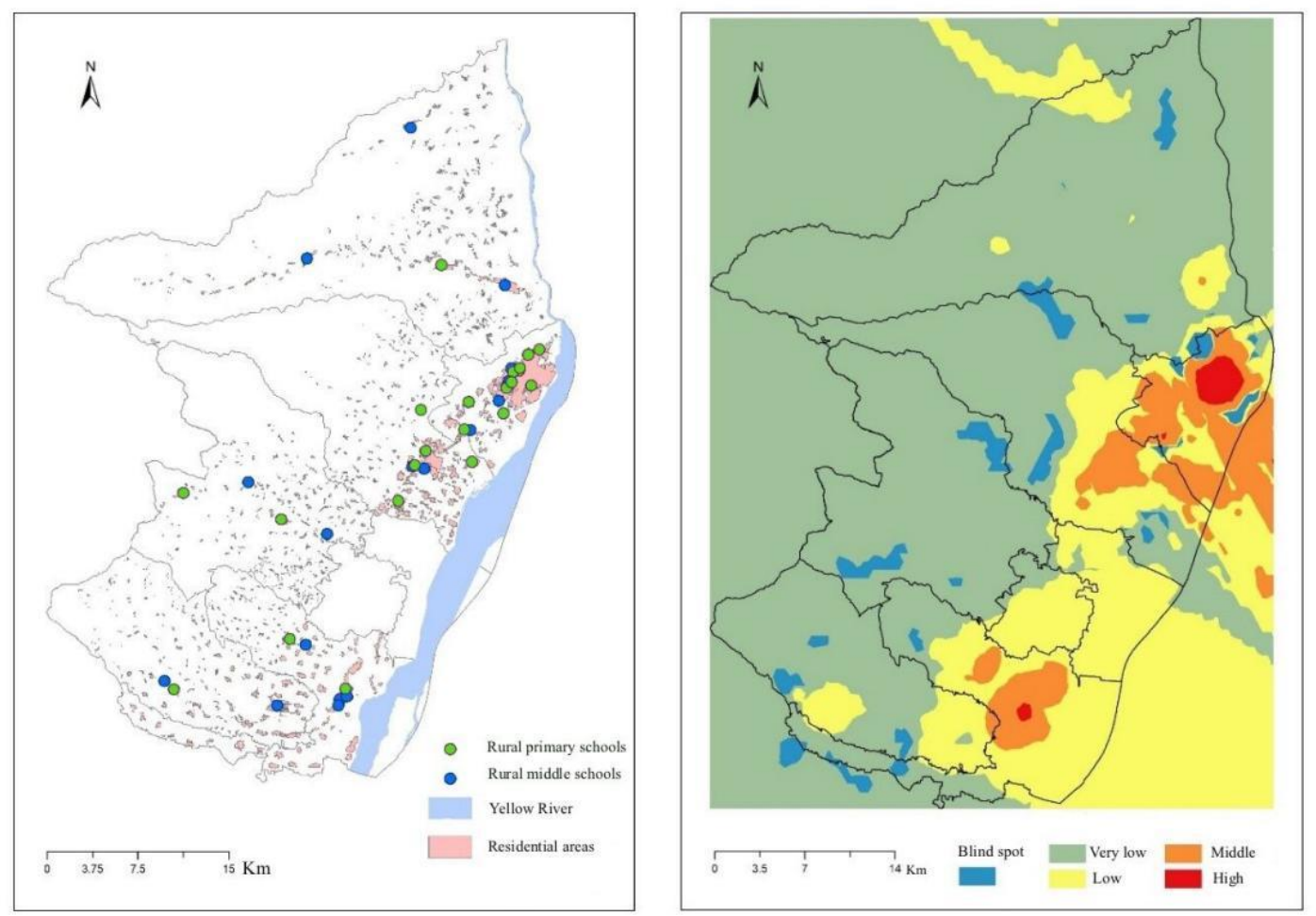

Figure 1. Schematic representation of the spatial coupling of population and schools

\section{Conclusion and discussion}

This study took 42 primary and secondary schools in six townships of Hancheng City and 2089 rural population clusters as research subjects. With the support of GIS technology and multiple data sources, based on network analysis, buffer zone analysis, population coupling model analysis, and spatial overlay analysis, the accessibility of primary and secondary education facilities and their spatial coupling with student population clusters were assessed at the administrative district scale and the small scale of rural 
population clusters. The results of the study help optimize the rational layout planning of rural primary and secondary schools, promote the exploration of scientific issues in educational geography, and are of great scientific value in promoting a balanced allocation of rural basic education resources, narrowing the urbanrural gap and achieving a balanced supply of basic public services in rural areas. The main research findings are as follows:

(1) The spatial imbalance in the current layout of education facilities in rural areas can be better identified from the perspective of small-scale demand agents. In the past, the planning of education facilities focused more on the total amount or per capita indicators but failed to consider the actual demand of the population and the level of supply of facilities on a spatial basis. There are relatively few smallscale studies on the optimization of the layout of educational resources at the level of population centers, i.e., residential areas. This paper takes the demand subject at the residential level as the starting point and finds that there is still a large degree of spatial "mismatch" between supply and demand while the demand for basic education in rural areas of Hancheng City remains unmet.

(2) The role of spatial barrier factors is obvious and that the conditions of the transportation network have an important influence on the optimization of the layout of rural primary and secondary schools. This phenomenon may be more prominent in the northwestern region, which is characterized by a wide distribution of mountainous terrain and a dotted distribution of the rural population, which is more dispersed and uneven than in the central and eastern rural areas of China; at the same time, the high ruggedness of rural roads in mountainous areas and the high construction costs lead to overall poor accessibility of roads. Improving transportation conditions can effectively increase the equality of opportunity for rural students to attend school near their homes, and also facilitate the rational allocation and use of teaching resources.

(3) Optimizing the spatial layout of primary and secondary schools by local conditions is an important way to achieve balanced development of basic education resources in rural areas of Hancheng City. For areas with better economic development and dense population and school, central school should be promoted as the management center and concentration of educational resources for the whole county, and boarding schools should be built to improve the equity of schooling for children in remote areas, such as Longmen Town. For areas such as Zhiyang Town and Zhichuan Town, where the student population is more dispersed, but the population catchment area is denser, the construction of rural teaching points should be given priority consideration. Teaching points facilitate remote students' access to schools nearby, save on education costs, provide equitable access to the same quality of education, and act as cultural agglomeration centers for small rural communities. Ideally, additional sites should be built evenly to cover the entire residential area, with priority given to areas with a dense distribution of settlements and reasonable road access if funds are scarce. In mountainous areas, which are often vast and sparsely populated, planning the layout of schools according to accessibility can result in a significant waste of public resources, so the quality of rural education can be improved by strengthening the construction of boarding schools and achieving a balanced allocation of resources between schools in the county. At the same time, an educationsharing model is being built, a system of exchange and rotation of headmasters and teachers within the county and a joint system of education between urban and rural areas is being built, and a mechanism of teacher mobility services is being established and improved to encourage a reasonable flow of teacher resources between urban and rural areas and between schools. This makes it possible for educational human and material resources to be used in a mobile manner between central schools and teaching points. In the current situation where rural educational resources are still in short supply, this concept should become a breakthrough in the balanced development of rural education. 
To realize the rural revitalization strategy, the construction of public service facilities in rural areas is an essential part of it, and the balanced spatial allocation of primary and secondary school education facilities is one of the top priorities. The construction of primary and secondary school education facilities in rural areas is a necessary step to coordinate urban and rural development, improve government functions, achieve equity and justice, and ensure that the nation shares the fruits of development. Based on the theories of effective spatial accessibility and balanced spatial supply and demand, this paper portrays the spatial allocation pattern of rural primary and secondary education facilities in Northwest China, and the research results have important theoretical significance and practical value for promoting the layout of urban and rural basic education resources in the context of rural revitalization strategies.

\section{Disclosure statement}

The authors declare no conflict of interest.

\section{References}

[1] Hansen WG, 1959, How Accessibility Shapes Land Use. J. Am. Plan. Assoc. https://doi.org/10.1080/01944365908978307

[2] Kelobonye K, Mao F, Xia J, et al., 2019, The Impact of Employment Self-Sufficiency Measures on Commuting Time: A Case Study of Perth, Australia. Sustainability, 11(5): 1488.

[3] Kelobonye K, Zhou H, McCarney G, et al., 2020, Measuring the Accessibility and Spatial Equity of Urban Services Under Competition Using the Cumulative Opportunities Measure," J. Transp. Geogr., 85: 102706.

[4] Song Z, 2010, Spatial Accessibility of Medical Services Based on Improved Potential Model and Determination of Areas with a Shortage of Medical Services: The Case of Rudong County, Jiangsu Province. Geoscience, 30(02): 213-219.

[5] Tao Z-L, Cheng Y, Dai T, 2014, Spatial Accessibility Evaluation of Elderly Facilities in Beijing. Advances in Geographical Sciences, 33(05): 616-624.

[6] Wang F, 2009, Quantitative Methods and Applications Based on GIS. The Commercial Press.

[7] Joseph AE, Phillips DR, 1984, Accessibility and Utilization: Geographical Perspectives on Health Care Delivery. Access. Util. Geogr. Perspect. Heal. Care Deliv. https://doi.org/10.2307/633309

[8] Zhang Y, 2016, Definition of Regional Spatial Equilibrium Connotation and State Assessment from the Perspective of Supply and Demand Drive - An Example from Shandong Province. Soft Science, 30(12): 54-58.

[9] Fan J, 2007, The Scientific Basis of the Zoning of the Main Functions in China. Journal of Geography, 2007(04): 339-350.

[10] Higgins B, Savoie DJ, Higgins B, et al., 2019, Interregional and International Trade, in Regional Development Theories \& Their Application.

[11] Allen T, Arkolakis C, 2014, Trade and the Topography of the Spatial Economy, Q. J. Econ. https://doi./org/10.1093/are/qju016

[12] Boyce MS, 2006, Scale for Resource Selection Functions. Diversity and Distributions, 12(3): 269-276.

[13] Holmes TJ, Fujita M, Krugman P, et al., 2000, The Spatial Economy: Cities, Regions, and International Trade. South. Econ. J., 67(2): 491-493. https://doi.org/10.2307/1061487 
[14] Ray ZK, Chen W, Sun W, 2008, A Study on Productivity Layout Based on the Concept of Spatial Equilibrium: The Case of Wuxi City. Geographical Research and Development, 2008(01): 19-22, 27.

[15] Lu DD, 2009, 2050: China’s Regional Development. Science Press.

[16] Chen W, Sun W, Zhao H, 2010, Spatial Imbalance Patterns and State Assessment of Regional Development: The Case of Jiangsu Province. Journal of Geography, 65(10): 1209-1217.

[17] Fan J, Zhou K, Chen D. 2013, Innovation and Application Practice of Economic Geography Research in Optimizing the Spatial Development Pattern of National Land in the Construction of Ecological Civilization. Economic Geography, 33(01): 1-8.

[18] Jing B, 2020, Study the Equilibrium State of the Human-Land Relationship and Spatial Control in the Qinba Mountains Based on the Supply-Demand Matching Model. Yangtze River Basin Resources and Environment, 29(03): 654-667.

[19] Song X, 2014, Exploration and improvement of urban primary and secondary school layout planning methods. Urban Planning, 38(08): 48-56.

[20] Yang X, Alain C, Song X, 2014, Limitations of Spatial Syntax in Urban Planning and Ways to Improve and Expand It. Journal of Urban Planning, 2014(05): 32-38.

[21] Song Y, 2015, Guide to the Application of GIS for Urban Spatial Analysis. China Construction Industry Press.

[22] Chen L, 2019, Study on the Spatial Matching of Urban Public Service Facilities and Residential Neighborhoods - A Case Study of Lanzhou City. Arid Zone Resources and Environment, 33(11): 120 127.

Publisher's note

Bio-Byword Scientific Publishing remains neutral with regard to jurisdictional claims in published maps and institutional affiliations. 